\title{
Extremal matrices for the Bruhat-graph order
}

\section{Rosário Fernandes \& Susana Furtado}

To cite this article: Rosário Fernandes \& Susana Furtado (2020): Extremal matrices for the Bruhat-graph order, Linear and Multilinear Algebra, DOI: 10.1080/03081087.2020.1749540

To link to this article: https://doi.org/10.1080/03081087.2020.1749540

\section{册 Published online: 07 Apr 2020.}

Submit your article to this journal 5

\section{山ll Article views: 5}

Q View related articles $\longleftarrow$

View Crossmark data \lceil 


\title{
Extremal matrices for the Bruhat-graph order
}

\author{
Rosário Fernandes ${ }^{a}$ and Susana Furtado ${ }^{b}$ \\ ${ }^{a}$ CMA and Departamento de Matemática da Faculdade de Ciências e Tecnologia, Universidade Nova de \\ Lisboa, Caparica, Portugal; ${ }^{b}$ CEAFEL and Faculdade de Economia do Porto, Universidade do Porto, Porto, \\ Portugal
}

\begin{abstract}
We consider the class $\mathcal{A}_{\text {sym }}^{0}(n, k)$ of symmetric $(0,1)$-matrices with zero trace and constant row sums $k$ which can be identified with the class of the adjacency matrices of $k$-regular undirected graphs. In a previous paper, two partial orders, the Bruhat and the Bruhatgraph order, have been introduced in this class. In fact, when $k=1$ or $k=2$, it was shown that the two orders coincide, while for $k \geq 3$ the two orders are distinct. In this paper we give general properties of minimal and maximal matrices for these orders on $\mathcal{A}_{\mathrm{sym}}^{0}(n, k)$ and study the minimal and maximal matrices when $k=1,2$ or 3 .
\end{abstract}

\section{ARTICLE HISTORY}

Received 24 May 2019

Accepted 15 March 2020

\section{COMMUNICATED BY}

J. Y. Shao

\section{KEYWORDS}

$(0,1)$-Matrices; Bruhat order; minimal matrices; maximal matrices; symmetric matrices

\section{AMS SUBJECT}

CLASSIFICATIONS

05B20; 06A07

\section{Introduction}

For any $n$-by- $n$ matrix $A=\left[a_{i, j}\right]$, let $\Sigma_{A}$ denote the $n$-by- $n$ matrix whose $(r, s)$-entry, with $1 \leq r, s \leq n$, is

$$
\sigma_{r, s}(A)=\sum_{i=1}^{r} \sum_{j=1}^{s} a_{i j} .
$$

Let $R=\left(r_{1}, \ldots, r_{n}\right)$ be a positive integral vector. We denote by $\mathcal{A}(R)$ the class of all $n$ by- $n(0,1)$-matrices whose row sum and column sum vectors are $R$. When $r_{i}=k$, for $i=1, \ldots, n$, we denote $\mathcal{A}(R)$ by $\mathcal{A}(n, k)$.

For $A_{1}, A_{2} \in \mathcal{A}(R)$, we say that $A_{1}$ precedes $A_{2}$ in the Bruhat order on $\mathcal{A}(R)$, and write $A_{1} \preceq_{B} A_{2}$, if, by the entrywise order, $\Sigma_{A_{1}} \geq \Sigma_{A_{2}}$. If $A_{1} \neq A_{2}$, we write $A_{1} \prec_{B} A_{2}$. The Bruhat order for $(0,1)$-matrices in the classes $\mathcal{A}(R)$ is a pre-order that has been extensively investigated [1-7].

In [3] the secondary Bruhat order was formally defined. For $A_{1}, A_{2} \in \mathcal{A}(R)$, we say that $A_{1}$ precedes $A_{2}$ in the secondary Bruhat order if $A_{1}$ is obtained from $A_{2}$ by a sequence of interchanges

$$
L_{2}=\left[\begin{array}{ll}
0 & 1 \\
1 & 0
\end{array}\right] \rightarrow I_{2}=\left[\begin{array}{ll}
1 & 0 \\
0 & 1
\end{array}\right],
$$

CONTACT Rosário Fernandes mrff@fct.unl.pt $\Theta$ CMA and Departamento de Matemática da Faculdade de Ciências e Tecnologia, Universidade Nova de Lisboa, 2829-516 Caparica, Portugal 
that replaces a submatrix of order 2 equal to $L_{2}$ by $I_{2}$.

It is easy to see that, if $A_{1}, A_{2} \in \mathcal{A}(R)$ and $A_{1}$ precedes $A_{2}$ in the secondary Bruhat order, then $A_{1} \preceq_{B} A_{2}$.

In [8], the authors studied the Bruhat and secondary Bruhat orders restricted to the class of $n$-by- $n$ symmetric $(0,1)$-matrices $A$ with zero trace and row sum vector $R$, denoted by $\mathcal{A}_{\text {sym }}^{0}(R)$. This class can be identified with the class of adjacency matrices of undirected graphs $G(A)$ with $n$ vertices and degree sequence $R$ (see [8] for details).

Let $A_{1}, A_{2} \in \mathcal{A}_{\text {sym }}^{0}(R)$. We say that $A_{1}$ is obtained from $A_{2}$ by a switch if $A_{1}$ and $A_{2}$ are equal except in a 4 -by-4 submatrix which is replaced by another matrix in three possible ways:

$$
\begin{aligned}
F_{S_{1}}:= & {\left[\begin{array}{llll}
0 & * & 0 & 1 \\
* & 0 & 1 & 0 \\
0 & 1 & 0 & * \\
1 & 0 & * & 0
\end{array}\right] \leftrightarrow\left[\begin{array}{llll}
0 & * & 1 & 0 \\
* & 0 & 0 & 1 \\
1 & 0 & 0 & * \\
0 & 1 & * & 0
\end{array}\right]=: B_{S_{1}} } \\
F_{S_{2}}:= & {\left[\begin{array}{llll}
0 & 0 & 1 & * \\
0 & 0 & * & 1 \\
1 & * & 0 & 0 \\
* & 1 & 0 & 0
\end{array}\right] \leftrightarrow\left[\begin{array}{llll}
0 & 1 & 0 & * \\
1 & 0 & * & 0 \\
0 & * & 0 & 1 \\
* & 0 & 1 & 0
\end{array}\right]=: B_{S_{2}} } \\
F_{S_{3}}:= & {\left[\begin{array}{llll}
0 & 0 & * & 1 \\
0 & 0 & 1 & * \\
* & 1 & 0 & 0 \\
1 & * & 0 & 0
\end{array}\right] \leftrightarrow\left[\begin{array}{llll}
0 & 1 & * & 0 \\
1 & 0 & 0 & * \\
* & 0 & 0 & 1 \\
0 & * & 1 & 0
\end{array}\right]=: B_{S_{3}}, }
\end{aligned}
$$

where $*$ denotes an unspecified entry. In each of (1), (2) and (3), we say that the matrix on the right is obtained from the matrix on the left by a forward switch, and the matrix on the left is obtained from the matrix on the right by a backward switch. The same terminology is used when these 4-by- 4 matrices are principal submatrices of $n$-by- $n$ symmetric matrices.

Let $A, C \in \mathcal{A}_{\text {sym }}^{0}(R)$, we say that $A$ precedes $C$ in the Bruhat-graph order, and we write $A \preceq_{B G} C$, if $A$ is obtained from $C$ by a sequence of forward switches. [8].

The Bruhat-graph order is the restriction of the secondary Bruhat order, on $\mathcal{A}_{\text {sym }}^{0}(R)$,

It follows easily that if $A_{1} \preceq_{B G} A_{2}$, then $A_{1} \preceq_{B} A_{2}$. In [8] the authors showed that the two orders coincide on $\mathcal{A}_{\text {sym }}^{0}(n, k)$, for $k=1,2$. We observe that if $k$ is odd and $\mathcal{A}_{\text {sym }}^{0}(n, k)$ is non-empty then $n$ is even.

In this paper we identify minimal and maximal matrices for the Bruhat-graph order on the classes $\mathcal{A}_{\text {sym }}^{0}(n, k)$ for $k=1,2,3$. We give a complete answer when $k=1,2$ and, in the case of minimal matrices, when $k=3$. In all cases the minimal matrices for the Bruhat and Bruhat-graph orders coincide. When $k=3$ we give a class of maximal matrices for the Bruhat-graph and Bruhat order and we conjecture that there are no maximal matrices beyond those.

The complementary class of $\mathcal{A}_{\text {sym }}^{0}(n, k)$ is the class $\mathcal{A}_{\text {sym }}^{0}(n, n-k-1)$. If $A \in$ $\mathcal{A}_{\text {sym }}^{0}(n, k)$, we write $A^{c}$ for the corresponding matrix $A^{c}=\left(J_{n}-I_{n}\right)-A$ in the complementary class $\mathcal{A}_{\text {sym }}^{0}(, n, n-k-1)$. If $A_{1}, A_{2} \in \mathcal{A}_{\text {sym }}^{0}(n, k)$ are such that $A_{1} \preceq_{B} A_{2}$ 
(respectively, $A_{1} \preceq_{B G} A_{2}$ ), then $A_{2}^{c} \preceq_{B} A_{1}^{c}$ (respectively, $A_{2}^{c} \preceq_{B G} A_{1}^{c}$ ). Thus the problem of identifying the minimal matrices in one of our classes is equivalent to identifying the maximal matrices in its complementary class.

The paper is organized as follows. In Section 2 we give some general properties of minimal and maximal matrices for the Bruhat-graph order (and Bruhat order) on $\mathcal{A}_{\text {sym }}^{0}(R)$ that will be crucial in identifying the extremal matrices in the classes we consider. In Sections 3-5 we consider the problem of identifying the minimal and maximal matrices in the classes $\mathcal{A}_{\text {sym }}^{0}(n, k)$ for $k=1,2,3$, respectively. Finally, in Section 6, we give some concluding remarks.

Here and throughout, we will use the following notation: given an $n$-by- $n$ matrix $A$, we denote the submatrix of $A$ indexed by rows $i_{1}, \ldots, i_{p}$ and columns $j_{1}, \ldots, j_{s}$ by $A\left[\left\{i_{1}, \ldots, i_{p}\right\} ;\left\{j_{1}, \ldots, j_{s}\right\}\right]$. If $\left\{i_{1}, \ldots, i_{p}\right\}=\left\{j_{1}, \ldots, j_{s}\right\}$, we simply write $A\left[\left\{i_{1}, \ldots, i_{p}\right\}\right]$.

For $i \geq 2$, we denote by $L_{i}$ the $i$-by- $i$ permutation matrix with 1 's on the antidiagonal and by $J_{i}$ the $i$-by- $i$ matrix with all entries equal to one.

\section{Properties of extremal matrices}

In this subsection we present some properties of the minimal and maximal matrices for the Bruhat-graph order on $\mathcal{A}_{\text {sym }}^{0}(R)$. Theorems 2.4 and 2.6 will be crucial in the proofs of the results in the rest of the paper.

We first make some observations that will be useful throughout.

Remark 2.1: If $A \in A_{\text {sym }}^{0}(R)$ is a minimal (resp. maximal) matrix for the Bruhat order on $A_{\text {sym }}^{0}(R)$, then $A$ is a minimal (resp. maximal) matrix for the Bruhat-graph order on $A_{\text {sym }}^{0}(R)$. Moreover, if $A$ is the unique minimal (resp. maximal) matrix for the Bruhatgraph order on $A_{\text {sym }}^{0}(R)$, then $A$ also is the unique minimal (resp. maximal) matrix for the Bruhat order on the same class.

Remark 2.2: Let $A \in A_{\text {sym }}^{0}(R)$ be an $n$-by- $n$ matrix, where $R$ is a positive integral vector of size $n$. Then, the following are equivalent:

- $A$ is a minimal matrix for the Bruhat-graph order on $A_{\text {sym }}^{0}(R)$;

- if $A[\{i, j\} ;\{k, l\}]=L_{2}$, with $1 \leq i<j \leq n$ and $1 \leq k<l \leq n$, then $i=k$ or $j=l$.

Recall that a minimal matrix for the Bruhat order on a class $\mathcal{A}(R)$ cannot contain a submatrix equal to $L_{2}$ [3].

The following technical result will be used in the proof of Theorem 2.4.

Lemma 2.3: Let $A=\left[a_{i j}\right]$ be a minimal matrix for the Bruhat-graph order on $\mathcal{A}_{\text {sym }}^{0}(R)$, where $R$ is a non-increasing positive integral vector of size $n$. Let $p, r, q$ be integers such that either $1 \leq p<q<r \leq n$ or $1 \leq q<r \leq p \leq n$. Let $b_{q}$ and $b_{r}$ be 0 if $p=1$ and, otherwise, be the sum of the columns of $A[\{1, \ldots, p-1\} ;\{q, r\}]$. If $b_{q} \leq b_{r}$ then $a_{p q}=1$ or $a_{p r}=0$.

Proof: Suppose that $a_{p q}=0$ and $a_{p r}=1$. Then $p \neq r$. Since $A$ has non-increasing column sums and $b_{q} \leq b_{r}$, there exists $j>p$ such that $a_{j q}=1$ and $a_{j r}=0$. Since $A[\{p, j\} ;\{q, r\}]=$ $L_{2}$ and $p \neq q$, by Remark 2.2 it follows that $r=j>p$, implying that $1 \leq p<q<r \leq n$. 
By symmetry, $a_{q r}=1$. Note that $a_{q q}=0$. But then, since $A$ has non-increasing column sums, there should exist $j^{\prime}>p$, with $j \neq j^{\prime}$, such that $a_{j^{\prime} q}=1$ and $a_{j^{\prime} r}=0$, implying that $A\left[\left\{p, j^{\prime}\right\} ;\{q, r\}\right]=L_{2}$, a contradiction.

Theorem 2.4: Let $A \in \mathcal{A}_{\text {sym }}^{0}(R)$ be a minimal matrix for the Bruhat-graph order on $\mathcal{A}_{\text {sym }}^{0}(R)$, where $R$ is a non-increasing positive integral vector of size $n$. Let $i$ be an integer with $1 \leq i \leq(n-1)$, and let $A_{i}$ be the $i$-by-n leading submatrix of $A$. For $k=1, \ldots, n$, let $b_{k}$ be the sum of the entries of the kth column of $A_{i}$. Then $b_{1} \geq \cdots \geq b_{i} \geq b_{i+1}-1$ and $b_{i+1} \geq \cdots \geq b_{n}$.

Proof: The proof is by induction on $i$. If $i=1$ the result follows easily from Lemma 2.3. Now suppose that $i>1$ and let $j$ be such that $1 \leq j<n$. Let $b_{j}^{(i-1)}$ and $b_{j+1}^{(i-1)}$ denote the sum of the entries in columns $j$ and $j+1$ of the $(i-1)$-by- $n$ leading submatrix of $A$. By the induction hypothesis, $b_{j}^{(i-1)} \geq b_{j+1}^{(i-1)}$ if $j \neq i-1$, and $b_{j}^{(i-1)} \geq b_{j+1}^{(i-1)}-1$ if $j=i-1$.

If $j \neq i-1, i$, using Lemma 2.3 in case $b_{j}^{(i-1)}=b_{j+1}^{(i-1)}$, it follows that $b_{j}^{(i)} \geq b_{j+1}^{(i)}$. The inequality $b_{i}^{(i)} \geq b_{i+1}^{(i)}-1$ follows because $b_{i}^{(i-1)} \geq b_{i+1}^{(i-1)}$. Now we show that $b_{i-1}^{(i)} \geq b_{i}^{(i)}$. If $i=2$ this inequality follows easily from Lemma 2.3. Suppose that $i>2$. By the induction hypothesis, $b_{i-1}^{(i-2)} \geq b_{i}^{(i-2)}$, where $b_{i-1}^{(i-2)}$ and $b_{i}^{(i-2)}$ denote the sum of the entries in columns $i-1$ and $i$ of the $(i-2)$-by- $n$ leading submatrix of $A$. Thus, using symmetry and the fact that the diagonal entries of $A$ are 0 , it follows that either $b_{i-1}^{(i)}=b_{i-1}^{(i-2)}$ and $b_{i}^{(i)}=b_{i}^{(i-2)}$, or $b_{i-1}^{(i)}=b_{i-1}^{(i-2)}+1$ and $b_{i}^{(i)}=b_{i}^{(i-2)}+1$. In any case, $b_{i-1}^{(i)} \geq b_{i}^{(i)}$.

Note that, in contrast with Theorem 2.4, the sum of the columns of any leading submatrix of a minimal matrix for the Bruhat order on $\mathcal{A}(R)$ [3], and on the class of symmetric matrices in $\mathcal{A}(R)$ [4], is non-increasing, where $R$ is a non-increasing positive integral vector.

We now focus on maximal matrices. We have an analogous of Remark 2.2.

Remark 2.5: Let $A \in \mathcal{A}_{\text {sym }}^{0}(R)$ be an $n$-by- $n$ matrix, where $R$ is a positive integral vector of size $n$. Then, the following are equivalent:

- $A$ is a maximal matrix for the Bruhat-graph order on $\mathcal{A}_{\text {sym }}^{0}(R)$;

- if $A[\{i, j\} ;\{k, l\}]=I_{2}$, with $1 \leq i<j \leq n$ and $1 \leq k<l \leq n$, then $i=l$ or $j=k$.

From Theorem 2.4 and the relation between maximal and minimal matrices described in the Introduction, we obtain the following result.

Theorem 2.6: Let $A \in \mathcal{A}_{\text {sym }}^{0}(R)$ be a maximal matrix for the Bruhat-graph order on $\mathcal{A}_{\mathrm{sym}}^{0}(R)$, where $R$ is a non-increasing positive integral vector of size $n>2$. Let $i=2, \ldots, n$ and let $A_{i}$ be the $i$-by-n leading submatrix of $A$. For $k=1, \ldots, n$, let $b_{k}$ be the sum of the entries of the kth column of $A_{i}$. Then, $b_{1} \leq \cdots \leq b_{i-2} \leq b_{i-1} \leq b_{i}+1$ and $b_{i} \leq \cdots \leq b_{n}$.

We now give some results regarding the construction of extremal matrices from smaller matrices. The next lemma, which can be easily proved, states that the direct sum of two 
minimal matrices for the Bruhat-graph order is a minimal matrix in the corresponding class.

Lemma 2.7: Let $R_{1}=\left(r_{11}, \ldots, r_{1 s_{1}}\right)$ and $R_{2}=\left(r_{21}, \ldots, r_{2 s_{2}}\right)$ be two non-increasing positive integral vectors such that $r_{1 s_{1}} \geq r_{21}$. Let $A \in \mathcal{A}_{\text {sym }}^{0}\left(R_{1}\right)$ and $C \in \mathcal{A}_{\text {sym }}^{0}\left(R_{2}\right)$. Then, the following are equivalent:

- $A \oplus C$ is a minimal matrix for the Bruhat-graph order on $\mathcal{A}_{\text {sym }}^{0}\left(\left(R_{1}, R_{2}\right)\right)$

- A is a minimal matrix for the Bruhat-graph order on $\mathcal{A}_{\text {sym }}^{0}\left(R_{1}\right)$ and $C$ is a minimal matrix for the Bruhat order on $\mathcal{A}_{\text {sym }}^{0}\left(R_{2}\right)$.

The next result provides another way of obtaining minimal matrices for the Bruhatgraph order on a class $\mathcal{A}_{\text {sym }}^{0}(R)$ from minimal matrices of smaller size.

Lemma 2.8: Let $R_{1}=\left(r_{11}, \ldots, r_{1 s_{1}}\right)$ and $R_{2}=\left(r_{21}, \ldots, r_{2 s_{2}}\right)$ be two vectors of non-negative integers and let $R=\left(r_{11}, \ldots, r_{1 s_{1}}+1, r_{21}+1, \ldots, r_{2 s_{2}}\right)$ be obtained by joining $R_{2}$ to $R_{1}$ after increasing $r_{1 s_{1}}$ and $r_{2 s_{2}}$ by 1 . If $A_{1}$ is a minimal matrix for the Bruhat-graph order on $\mathcal{A}_{\text {sym }}^{0}\left(R_{1}\right)$ and $A_{2}$ is a minimal matrix for the Bruhat-graph order on $\mathcal{A}_{\text {sym }}^{0}\left(R_{2}\right)$, then

$$
A=\left[\begin{array}{ll}
A_{1} & A_{3} \\
A_{3}^{T} & A_{2}
\end{array}\right], \text { where } A_{3}=\left[\begin{array}{cccc}
0 & 0 & \cdots & 0 \\
\vdots & \vdots & & \vdots \\
0 & 0 & \cdots & 0 \\
1 & 0 & \cdots & 0
\end{array}\right]
$$

is a minimal matrix for the Bruhat-graph order on $\mathcal{A}_{\mathrm{sym}}^{0}(R)$.

Proof: The result follows by Remark 2.2 noting that the only submatrix $L_{2}$ of $A$ not contained in $A_{1}$ nor in $A_{2}$ is the one lying in rows and columns $s_{1}, s_{1}+1$.

Let $C$ and $D$ be $k$-by- $k$ and $l$-by- $l$ matrices, respectively. Then their skew-direct sum is defined as the $(k+l) \times(k+l)$-matrix

$$
C \oplus^{\prime} D:=\left[\begin{array}{c|c}
O_{k l} & C \\
\hline D & O_{l k}
\end{array}\right] .
$$

Taking into account Remark 2.5 we obtain the following.

Lemma 2.9: Let $A \in \mathcal{A}\left(n_{1}, k\right)$ and $C \in \mathcal{A}_{\text {sym }}^{0}\left(n_{2}, k\right)$. Then, the following are equivalent:

- $A$ is a maximal matrix for the secondary Bruhat order on $\mathcal{A}\left(n_{2}, k\right)$ and $C$ is a maximal matrix for the Bruhat-graph order on $\mathcal{A}_{\text {sym }}^{0}\left(n_{2}, k\right)$

- $A^{T} \oplus^{\prime} C \oplus^{\prime} A$ is a maximal matrix for the Bruhat-graph order on $\mathcal{A}_{\text {sym }}^{0}\left(2 n_{1}+n_{2}, k\right)$.

Remark 2.10: Using the definition of $\preceq_{B}$, it can be easily verified that Lemmas 2.7, 2.8 and 2.9 remain valid if Bruhat-graph order and secondary Bruhat order are replaced by Bruhat order. 


\section{Extremal matrices on $\mathcal{A}_{\text {sym }}^{0}(n, 1)$}

A matrix $A \in \mathcal{A}_{\text {sym }}^{0}(n, 1)$ is a symmetric permutation matrix of order $n$ with zero trace. Hence $n$ is even, $A$ is the adjacency matrix of the union of the complete graphs $K_{2}$, and $A$ is a product of disjoint transpositions.

In [8], it is shown that the Bruhat and Bruhat-graph order coincide on the class $\mathcal{A}_{\text {sym }}^{0}(n, 1)$.

Theorem 3.1: Let $n \geq 2$ be an even integer. Then $L:=L_{2} \oplus L_{2} \oplus \cdots \oplus L_{2}$ is the unique minimal matrix for the Bruhat order on $\mathcal{A}_{\mathrm{sym}}^{0}(n, 1)$, and $L_{n}$ is the unique maximal matrix.

Proof: Let $B \in \mathcal{A}_{\text {sym }}^{0}(n, 1)$. Let $i, j \in\{1, \ldots, n\}$. If either $i \neq j$ or $i=j$ is even, then $\sigma_{i j}(L)=$ $\min \{i, j\}$ and, thus, $\sigma_{i j}(B) \leq \sigma_{i j}(L)$. If $i=j$ is odd, then $\sigma_{i j}(L)=i-1$, which, since $i$ is odd, is the maximum number of 1's possible for an $i$-by- $i$ principal submatrix of a matrix in $\mathcal{A}_{\text {sym }}^{0}(n, 1)$. Thus, in this case we also have $\sigma_{i i}(B) \leq \sigma_{i i}(L)$. We then have $L \preceq_{B} B$. Hence, $L$ is the unique minimal matrix.

In [3] it was mentioned that $J_{n}-L_{n}$ is the unique minimal matrix in $\mathcal{A}(n, n-1)$. From a comment in [1], it follows that $L_{n}$ is the unique maximal matrix for the Bruhat order on $\mathcal{A}(n, 1)$. Since $L_{n} \in \mathcal{A}_{\text {sym }}^{0}(n, 1)$, it follows that $L_{n}$ is the unique maximal matrix.

\section{Extremal matrices on $\mathcal{A}_{\text {sym }}^{0}(n, 2)$}

A matrix $A \in \mathcal{A}_{\text {sym }}^{0}(n, 2)$ is the adjacency matrix of the union of cycles. If $n=3$, then $\mathcal{A}_{\text {sym }}(3,2)$ contains a unique matrix, which is the adjacency matrix of a cycle with 3 vertices. A matrix in $\mathcal{A}_{\text {sym }}^{0}(4,2)$ is the adjacency matrix of a cycle with 4 vertices.

In [8], it is shown that the Bruhat and Bruhat-graph order coincide on the class $\mathcal{A}_{\text {sym }}^{0}(n, 2)$.

\subsection{Minimal matrices}

The next result describes the minimal matrices for the Bruhat order on $\mathcal{A}_{\text {sym }}^{0}(n, 2)$. In particular, it follows that for $3 \leq n \leq 6$, there is a unique minimal matrix for the Bruhat order on $\mathcal{A}_{\text {sym }}^{0}(n, 2)$.

Theorem 4.1: Let $n \geq 3$ be an integer. A matrix in $\mathcal{A}_{\mathrm{sym}}^{0}(n, 2)$ is a minimal matrix for the Bruhat-graph order on $\mathcal{A}_{\mathrm{sym}}^{0}(n, 2)$ if and only if it is a direct sum of the matrices

$$
M_{1}=\left[\begin{array}{lll}
0 & 1 & 1 \\
1 & 0 & 1 \\
1 & 1 & 0
\end{array}\right], \quad M_{2}=\left[\begin{array}{llll}
0 & 1 & 1 & 0 \\
1 & 0 & 0 & 1 \\
1 & 0 & 0 & 1 \\
0 & 1 & 1 & 0
\end{array}\right], \quad M_{3}=\left[\begin{array}{lllll}
0 & 1 & 1 & 0 & 0 \\
1 & 0 & 0 & 1 & 0 \\
1 & 0 & 0 & 0 & 1 \\
0 & 1 & 0 & 0 & 1 \\
0 & 0 & 1 & 1 & 0
\end{array}\right] \text {. }
$$

Proof: The matrices $M_{1}, M_{2}$ and $M_{3}$ are the unique matrices that are obtained by application of Remark 2.2 and Theorem 2.4 and that cannot be written as direct sums of smaller matrices. By Remark 2.2, they are minimal for the Bruhat-graph order. Now the claim follows from Lemma 2.7 . 
Note that any integer $n \geq 3$ can be written as $3 p+4 q+5 r$, for some non-negative integers $p, q, r$.

\subsection{Maximal matrices}

We next describe the maximal matrices for the Bruhat order on $\mathcal{A}_{\mathrm{sym}}^{0}(n, 2)$.

Theorem 4.2: Let $n \geq 3$ be an integer. A matrix in $\mathcal{A}_{\text {sym }}^{0}(n, 2)$ is a maximal matrix for the Bruhat-graph order on $\mathcal{A}_{\mathrm{sym}}^{0}(n, 2)$ if and only if it is of the form

$$
A_{1} \oplus^{\prime} \cdots \oplus^{\prime} A_{r} \oplus^{\prime} A_{r} \oplus^{\prime} \cdots \oplus^{\prime} A_{1}, \quad r \geq 1
$$

or

$$
A_{1} \oplus^{\prime} \cdots \oplus^{\prime} A_{r} \oplus^{\prime} B \oplus^{\prime} A_{r} \oplus^{\prime} \cdots \oplus^{\prime} A_{1}, \quad r \geq 0,
$$

where $A_{i} \in\left\{M_{1}, J_{2}\right\}, i=1, \ldots, r$ and $B \in\left\{M_{1}, M_{4}\right\}$, with

$$
M_{1}=\left[\begin{array}{lll}
0 & 1 & 1 \\
1 & 0 & 1 \\
1 & 1 & 0
\end{array}\right] \text { and } M_{4}=\left[\begin{array}{lllll}
0 & 0 & 0 & 1 & 1 \\
0 & 0 & 1 & 0 & 1 \\
0 & 1 & 0 & 1 & 0 \\
1 & 0 & 1 & 0 & 0 \\
1 & 1 & 0 & 0 & 0
\end{array}\right] .
$$

Proof: By Remark 2.5, the matrices $M_{1}$ and $M_{4}$ are maximal for the Bruhat-graph order. On the other hand, the matrix $J_{2}$ and $M_{1}$ are maximal for the secondary Bruhat order. Using Lemma 2.9, the claimed skew-direct sum of them is maximal for the Bruhat-graph order.

Conversely, suppose that $A$ is a maximal matrix for the Bruhat order on $\mathcal{A}_{\text {sym }}^{0}(n, 2)$. Then, by Theorem 2.6, $A$ has the form

$$
A=\left[\begin{array}{ccccccc}
0 & 0 & 0 & \cdots & 0 & 1 & 1 \\
0 & 0 & & & & & \\
0 & & & & & & \\
1 & & & & & & \\
1 & & & & & &
\end{array}\right] .
$$

Case (1) Suppose that $a_{2, n}=1$.

Case (1.1) Suppose that $a_{2, n-1}=1$. Thus, $A=J_{2} \oplus^{\prime} A^{\prime} \oplus^{\prime} J_{2}$, for some (possibly empty) matrix $A^{\prime}$ which, by Theorem 2.9, is maximal for the Bruhat order on $\mathcal{A}_{\text {sym }}^{0}(n-4,2)$.

Case (1.2) Suppose that $a_{2, n-1}=0$. If $n=3$, then $A=M_{1}$. Now suppose that $n>3$. Then

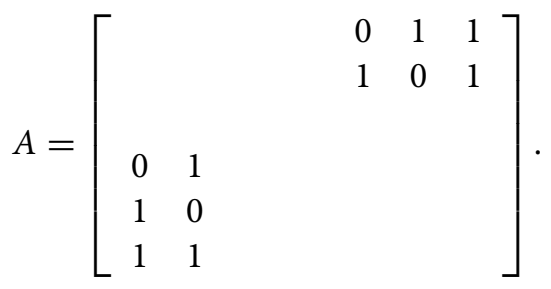


Case (1.2.1) Suppose that $a_{3, n-1}=1$. If $n=5$ then

$$
A=\left[\begin{array}{lllll}
0 & 0 & 0 & 1 & 1 \\
0 & 0 & 1 & 0 & 1 \\
0 & 1 & 0 & 1 & 0 \\
1 & 0 & 1 & 0 & 0 \\
1 & 1 & 0 & 0 & 0
\end{array}\right]=M_{4}
$$

If $n>5$ then $a_{3, n-2}=1$, otherwise $A[\{2,3, n-2, n-1\}]$ would have the form $B_{S_{1}}$. Thus,

$$
A=\left[\begin{array}{lll}
0 & 1 & 1 \\
1 & 0 & 1 \\
1 & 1 & 0
\end{array}\right] \oplus^{\prime} A^{\prime} \oplus^{\prime}\left[\begin{array}{lll}
0 & 1 & 1 \\
1 & 0 & 1 \\
1 & 1 & 0
\end{array}\right]=M_{1} \oplus^{\prime} A^{\prime} \oplus^{\prime} M_{1},
$$

for some (possibly empty) matrix $A^{\prime}$ which, by Theorem 2.9, is maximal for the Bruhat order on $\mathcal{A}_{\text {sym }}^{0}(n-6,2)$.

Case (1.2.2) Suppose that $a_{3, n-1}=0$. Then, $n>6$ and

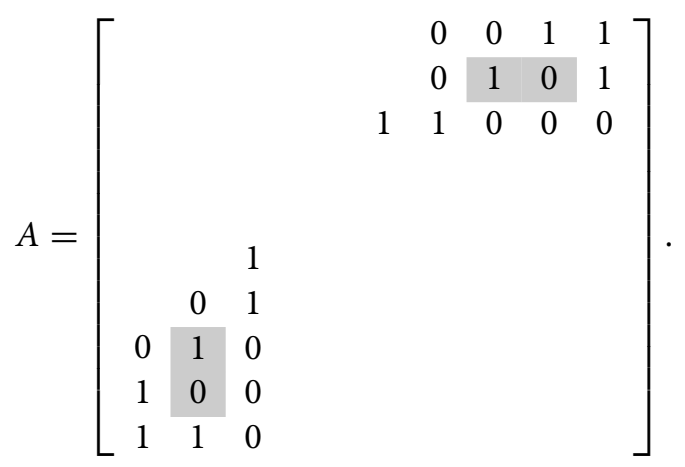

Therefore, there is $p>3$ such that $a_{p, n-1}=1$. If $p \neq n-2$, then $a_{p, n-2}=1$, as otherwise $A[\{2, p, n-2, n-1\}]$ would have the form $B_{S_{1}}$. Analogously, if $p \neq n-3$, then $a_{p, n-3}=$ 1 , and if $p \neq n-4$, then $a_{p, n-4}=1$. Then row $p$ has more than 2 ones, a contradiction.

Case (2) Suppose that $a_{2, n}=0$. Then, $n>5$ and

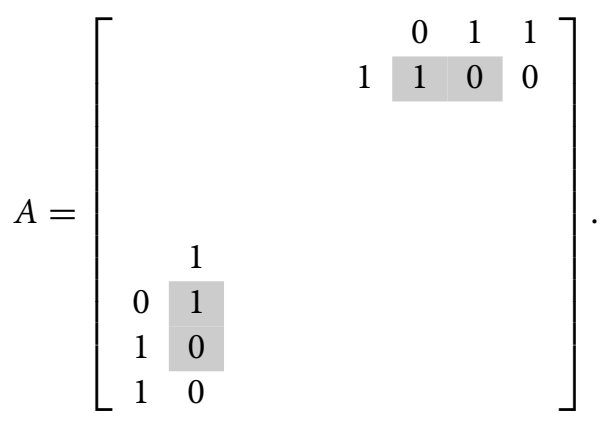

Then, there is $p>2$ such that $a_{p, n-1}=1$. If $p \neq n-2$, then $a_{p, n-2}=1$, as otherwise $A[\{2, p, n-2, n-1\}]$ would have the form $B_{S_{1}}$. Analogously, if $p \neq n-3$, then $a_{p, n-3}=$ 1. Since row $p$ has exactly 2 ones, it follows that $p \in\{n-2, n-3\}$. If $p=n-2$ then $a_{p, n-3}=1$. Since $a_{p, n-1}=1$ and $a_{p, 2}=a_{2, p}=1$, row $p$ would have more than 2 ones, 
a contradiction. Analogously, if $p=n-3$ then $a_{p, n-2}=1$. Since $a_{p, n-1}=1$ and $a_{p, 2}=$ $a_{2, p}=1$, row $p$ would have more than 2 ones, a contradiction.

Example 4.3: Let $n=8$. Then $n=3+5=4+4$. By Theorem 4.1 there are then three minimal matrices for the Bruhat order on $\mathcal{A}_{\text {sym }}^{0}(8,2): M_{2} \oplus M_{2}, M_{1} \oplus M_{3}$ and $M_{3} \oplus M_{1}$. There is a unique maximal matrix for the Bruhat order on $A_{\text {sym }}^{0}(8,2)$, namely

$$
D=J_{2} \oplus^{\prime} J_{2} \oplus^{\prime} J_{2} \oplus^{\prime} J_{2}
$$

In fact, the matrix $C=J_{2} \oplus J_{2} \oplus J_{2} \oplus J_{2}$ is the unique minimal matrix for the Bruhat order on $\mathcal{A}(8,2)$ [2]. Thus, $L_{8} C=D$ is the unique maximal matrix on $\mathcal{A}(8,2)$ and hence the unique maximal matrix on $\mathcal{A}_{\text {sym }}^{0}(8,2)$.

\section{Extremal matrices on $\mathcal{A}_{\text {sym }}^{0}(n, 3)$}

In this section we describe the minimal matrices and a class of maximal matrices for the Bruhat-graph order on $\mathcal{A}_{\text {sym }}^{0}(n, 3), n \geq 4$. Note that in this case $n$ is even.

\subsection{Minimal matrices}

In Theorem 5.3, we describe the minimal matrices for the Bruhat-graph order on $\mathcal{A}_{\text {sym }}^{0}(n, 3)$. We make use of the following six matrices:

$$
\begin{gathered}
X=\left[\begin{array}{llll}
0 & 1 & 1 & 1 \\
1 & 0 & 1 & 1 \\
1 & 1 & 0 & 1 \\
1 & 1 & 1 & 0
\end{array}\right], \quad Y=\left[\begin{array}{llllll}
0 & 1 & 1 & 1 & 0 & 0 \\
1 & 0 & 1 & 0 & 1 & 0 \\
1 & 1 & 0 & 0 & 0 & 1 \\
1 & 0 & 0 & 0 & 1 & 1 \\
0 & 1 & 0 & 1 & 0 & 1 \\
0 & 0 & 1 & 1 & 1 & 0
\end{array}\right], \\
H=\left[\begin{array}{llllll}
0 & 1 & 1 & 0 & 0 & 0 \\
1 & 0 & 1 & 1 & 0 & 0 \\
1 & 1 & 0 & 0 & 1 & 0 \\
0 & 1 & 0 & 0 & 1 & 1 \\
0 & 0 & 1 & 1 & 0 & 1 \\
0 & 0 & 0 & 1 & 1 & 0
\end{array}\right], \quad \begin{array}{llll} 
\\
E
\end{array}=\left[\begin{array}{lllllll}
0 & 1 & 1 & 1 & 0 & 0 & 0 \\
1 & 0 & 1 & 1 & 0 & 0 & 0 \\
1 & 1 & 0 & 0 & 1 & 0 & 0 \\
1 & 1 & 0 & 0 & 0 & 1 & 0 \\
0 & 0 & 1 & 0 & 0 & 1 & 1 \\
0 & 0 & 0 & 1 & 1 & 0 & 1 \\
0 & 0 & 0 & 0 & 1 & 1 & 0
\end{array}\right], \quad F=\left[\begin{array}{llllll}
0 & 1 & 1 & 1 & 0 \\
1 & 0 & 1 & 1 & 0 \\
1 & 1 & 0 & 0 & 1 \\
1 & 1 & 0 & 0 & 1 \\
0 & 0 & 1 & 1 & 0
\end{array}\right],
\end{gathered}
$$




$$
G=\left[\begin{array}{llll}
0 & 1 & 1 & 0 \\
1 & 0 & 1 & 1 \\
1 & 1 & 0 & 1 \\
0 & 1 & 1 & 0
\end{array}\right]
$$

We also consider the $i$-by- $i$ matrices

$$
K_{i}=\left[\begin{array}{cccc}
0 & 0 & \cdots & 0 \\
\vdots & \vdots & & \vdots \\
0 & 0 & \cdots & 0 \\
1 & 0 & \cdots & 0
\end{array}\right]
$$

If the order of $K_{i}$ is clear from the context, we write just $K$ instead of $K_{i}$.

Proposition 5.1: The matrices $X, Y, E, F, G$ and $H$ are minimal for the Bruhat order, and, therefore, for the Bruhat-graph order, on their corresponding classes $\mathcal{A}_{\text {sym }}^{0}(R)$.

Proof: The result can be easily verified by assuming that, for each $A \in\{X, Y, E, F, G, H\}$, there is a matrix $B \in \mathcal{A}_{\text {sym }}^{0}(R)$ such that $B \prec_{B} A$ and using the definition of $\prec_{B}$ in order to get a contradiction.

To characterize the minimal matrices in $\mathcal{A}_{\text {sym }}^{0}(n, 3)$, we first prove the following lemma.

Lemma 5.2: Let $A \in \mathcal{A}_{\mathrm{sym}}^{0}(n, 3)$ be a minimal matrix for the Bruhat-graph order on $\mathcal{A}_{\text {sym }}^{0}(n, 3)$ of the form

$$
A=\left[\begin{array}{ll}
A_{1} & K \\
K^{T} & B
\end{array}\right],
$$

where $A_{1}$ is square. Then either $B=B_{1} \oplus B_{2}$, with $B_{1} \in\left\{L_{7} E L_{7}, L_{5} F L_{5}\right\}$ ( $B_{2}$ may be empty), or

$$
B=\left[\begin{array}{ll}
C_{1} & K \\
K^{T} & C_{2}
\end{array}\right]
$$

with $C_{1} \in\{G, H\}$.

Proof: Suppose that $A \in \mathcal{A}_{\text {sym }}^{0}(n, 3)$ is a minimal matrix for the Bruhat-graph order on $\mathcal{A}_{\text {sym }}^{0}(n, 3)$ as in (4). Let $B=\left[b_{i j}\right]$. By Theorem 2.4 and symmetry,

$$
B=\left[\begin{array}{llll}
0 & 1 & 1 & 0 \\
1 & 0 & & \\
1 & & & \\
0 & & &
\end{array}\right] .
$$

Case (1) Suppose that $b_{23}=0$. 
Case (1.1) Suppose that $b_{34}=0$. Then, by Theorem 2.4,

$$
B=\left[\begin{array}{lllllllll}
0 & 1 & 1 & & & & \\
1 & 0 & 0 & 1 & 1 & & \\
1 & 0 & 0 & 0 & 0 & 1 & 1 \\
& 1 & 0 & 0 & & & \\
0 & 1 & 0 & & & & \\
& & 1 & & & & \\
& & 1 & & & &
\end{array}\right]
$$

Moreover, $b_{4,6}=b_{4,7}=b_{5,6}=b_{5,7}=1$, as otherwise, for $i \in\{4,5\}, j \in\{6,7\}, B[\{2,3, i, j\}]$ would have the form $F_{S_{2}}$. Thus,

$$
B=\left[\begin{array}{llllllll}
0 & 1 & 1 & & & & \\
1 & 0 & 0 & 1 & 1 & & \\
1 & 0 & 0 & 0 & 0 & 1 & 1 \\
& 1 & 0 & 0 & 0 & 1 & 1 \\
0 & 1 & 0 & 0 & 0 & 1 & 1 \\
& & 1 & 1 & 1 & 0 & 0 \\
& & 1 & 1 & 1 & 0 & 0
\end{array}\right]
$$

a contradiction since $B[\{4,5,6,7\}]$ has the form $F_{S_{2}}$.

Case (1.2) Suppose that $b_{34}=1$.

Case (1.2.1) Suppose that $b_{3,5}=1$. Then, by Theorem 2.4,

$$
B=\left[\begin{array}{llllll}
0 & 1 & 1 & & \\
1 & 0 & 0 & 1 & 1 \\
1 & 0 & 0 & 1 & 1 \\
& 1 & 1 & 0 & \\
0 & 1 & 1 & & 0
\end{array}\right]
$$

Note that $b_{45}=1$, otherwise $B[\{2,3,4,5\}]$ has the form $F_{S_{2}}$. Thus, by symmetry, it follows that $B=\left(L_{5} F L_{5}\right) \oplus B_{2}$, for some matrix $B_{2}$ (possibly empty).

Case (1.2.2) Suppose that $b_{3,5}=0$. Then, by Theorem 2.4,

$$
B=\left[\begin{array}{lllllll}
0 & 1 & 1 & & & \\
1 & 0 & 0 & 1 & 1 & \\
1 & 0 & 0 & 1 & 0 & 1 \\
& 1 & 1 & 0 & & \\
0 & 1 & 0 & & & \\
& & 1 & & &
\end{array}\right]
$$


Moreover, $b_{46}=1$, as otherwise $B[\{2,3,4,6\}]$ has the form $F_{S_{2}}$. Thus,

$$
B=\left[\begin{array}{lllllll}
0 & 1 & 1 & & & \\
1 & 0 & 0 & 1 & 1 & \\
1 & 0 & 0 & 1 & 0 & 1 \\
& 1 & 1 & 0 & 0 & 1 \\
0 & 1 & 0 & 0 & 0 & \\
& & 1 & 1 & & 0
\end{array}\right]
$$

a contradiction since $B[\{2,3,4,5\}]$ has the form $F_{S_{3}}$.

Case (2) Suppose that $b_{23}=1$. We have, by Theorem 2.4,

$$
B=\left[\begin{array}{llll}
0 & 1 & 1 & \\
1 & 0 & 1 & 1 \\
1 & 1 & 0 & \\
& 1 & &
\end{array}\right]
$$

Case (2.1) Suppose that $b_{34}=1$. Then

$$
B=\left[\begin{array}{lllll}
0 & 1 & 1 & & \\
1 & 0 & 1 & 1 & \\
1 & 1 & 0 & 1 & \\
& 1 & 1 & 0 & 1 \\
& & & 1 &
\end{array}\right]=\left[\begin{array}{cc}
G & K \\
K^{T} & C_{2}
\end{array}\right]
$$

for some matrix $C_{2}$.

Case (2.2) Suppose that $b_{34}=0$. Then $b_{46}=1$. Moreover, $b_{56}=1$, otherwise $B[\{3,4,5,6\}]$ has the form $F_{S_{2}}$. Thus,

$$
B=\left[\begin{array}{lllllll}
0 & 1 & 1 & & & \\
1 & 0 & 1 & 1 & 0 & \\
1 & 1 & 0 & 0 & 1 & \\
& 1 & 0 & 0 & & 1 \\
& 0 & 1 & & 0 & 1 \\
& & & 1 & 1 & 0
\end{array}\right]
$$

Case (2.2.1) Suppose that $b_{45}=0$. Then

$$
B=\left[\begin{array}{llllllll}
0 & 1 & 1 & & & & \\
1 & 0 & 1 & 1 & 0 & & \\
1 & 1 & 0 & 0 & 1 & & \\
& 1 & 0 & 0 & 0 & 1 & 1 \\
& 0 & 1 & 0 & 0 & 1 & 1 \\
& & & 1 & 1 & 0 & \\
& & & 1 & 1 & & 0
\end{array}\right]
$$


We have $b_{76}=1$, otherwise $B[\{4,5,6,7\}]$ has the form $F_{S_{2}}$. Thus, $B=L_{7} E L_{7} \oplus B_{2}$, for some matrix $B_{2}$ (possibly empty).

Case (2.2.2) Suppose that $b_{45}=1$. Then $b_{56}=1$, otherwise $B[\{3,4,5,6\}]$ has the form $F_{S_{2}}$. Thus,

$$
B=\left[\begin{array}{lllllll}
0 & 1 & 1 & & & & \\
1 & 0 & 1 & 1 & 0 & & \\
1 & 1 & 0 & 0 & 1 & & \\
& 1 & 0 & 0 & 1 & 1 & \\
& 0 & 1 & 1 & 0 & 1 & \\
& & & 1 & 1 & 0 & 1 \\
& & & & & 1 &
\end{array}\right]=\left[\begin{array}{cc}
H & K \\
K^{T} & C_{2}
\end{array}\right]
$$

for some matrix $C_{2}$.

Theorem 5.3: Let $n \geq 4$ be an even integer. Then, a matrix in $\mathcal{A}_{\mathrm{sym}}^{0}(n, 3)$ is a minimal matrix for the Bruhat-graph order on $\mathcal{A}_{\mathrm{sym}}^{0}(n, 3)$ if and only if it is a direct sum of the matrices $X, Y$, and

$$
Z\left(B_{1}, C_{1}, \ldots, C_{p}, B_{2}\right)=\left[\begin{array}{cccccc}
B_{1} & K & & & & 0 \\
K^{T} & C_{1} & K & & & \\
& K^{T} & C_{2} & \ddots & & \\
& & \ddots & \ddots & K & \\
& & & K^{T} & C_{p} & K \\
0 & & & & K^{T} & B_{2}
\end{array}\right], \quad p \geq 0
$$

where $B_{1} \in\{E, F\}, B_{2} \in\left\{L_{7} E L_{7}, L_{5} F L_{5}\right\}, C_{i} \in\{G, H\}, i=1, \ldots, p$.

Proof: If $A$ is a matrix of the claimed form, then by Proposition 5.1, Lemmas 2.7 and 2.8, it follows that $A$ is minimal in $\mathcal{A}_{\text {sym }}^{0}(n, 3)$.

Now we prove the converse. Let $A=\left[a_{i, j}\right]$ be a minimal matrix for the Bruhat order on $\mathcal{A}_{\text {sym }}^{0}(n, 3)$. By Theorem 2.4, $A$ has the form 
Case (1) Suppose that $a_{2,3}=0$. By Theorem 2.4 and symmetry,

$$
A=\left[\begin{array}{llllll}
0 & 1 & 1 & 1 & 0 & \\
1 & 0 & 0 & 0 & 1 & 1 \\
1 & 0 & 0 & & & \\
1 & 0 & & & & \\
0 & 1 & & & & \\
& 1 & & & &
\end{array}\right]
$$

By Theorem 2.4, we have $a_{43}=0$ and, by symmetry, $a_{34}=0$. Then, using similar arguments, we obtain

$$
A=\left[\begin{array}{lllllllll}
0 & 1 & 1 & 1 & 0 & & & \\
1 & 0 & 0 & 0 & 1 & 1 & & \\
1 & 0 & 0 & 0 & 0 & 0 & 1 & 1 \\
1 & 0 & 0 & 0 & & & & \\
0 & 1 & 0 & & & & & \\
& 1 & 0 & & & & & \\
& & 1 & & & & & \\
& & 1 & & & & &
\end{array}\right]
$$

We have $a_{7,5}=1$ and $a_{7,6}=1$, otherwise $A[\{2,3,5,7\}]$ or $A\left[\{2,3,6,7\}\right.$ has the form $F_{S_{2}}$. Similarly, $a_{8,5}=1$ and $a_{8,6}=1$, otherwise $A[\{2,3,5,8\}]$ or $A[\{2,3,6,8\}]$ has the form $F_{S_{2}}$. Therefore, by symmetry,

$$
A=\left[\begin{array}{llllllll}
0 & 1 & 1 & 1 & 0 & & & \\
1 & 0 & 0 & 0 & 1 & 1 & & \\
1 & 0 & 0 & 0 & 0 & 0 & 1 & 1 \\
1 & 0 & 0 & 0 & & & & \\
0 & 1 & 0 & & 0 & & 1 & 1 \\
0 & 1 & 0 & & & 0 & 1 & 1 \\
0 & 0 & 1 & & 1 & 1 & 0 & \\
0 & 0 & 1 & & 1 & 1 & & 0
\end{array}\right]
$$

Since rows 5 to 8 and column 5 already have three 1's, there exists $l>8$ such that $a_{l 4}=1$ and $a_{l 5}=0$. Then $A[\{2,4,5, l\}]$ has the form $F_{S_{2}}$.

Case (2) Suppose that $a_{2,3}=1$. Then,

$$
A=\left[\begin{array}{lllll}
0 & 1 & 1 & 1 & 0 \\
1 & 0 & 1 & & \\
1 & 1 & 0 & & \\
1 & & & & \\
0 & & & &
\end{array}\right]
$$

Case (2.1) Suppose that $a_{24}=1$. 
Case (2.1.1) Suppose that $a_{34}=1$. Then, using symmetry,

$$
A=\left[\begin{array}{llll}
0 & 1 & 1 & 1 \\
1 & 0 & 1 & 1 \\
1 & 1 & 0 & 1 \\
1 & 1 & 1 & 0
\end{array}\right] \oplus A^{\prime}=X \oplus A^{\prime}
$$

for some $A^{\prime} \in \mathcal{A}_{\text {sym }}^{0}(n-4,3)$.

Case (2.1.2) Suppose that $a_{34}=0$. Then, by Theorem 2.4 and symmetry,

$$
A=\left[\begin{array}{lllll}
0 & 1 & 1 & 1 & 0 \\
1 & 0 & 1 & 1 & 0 \\
1 & 1 & 0 & 0 & 1 \\
1 & 1 & 0 & 0 & \\
0 & 0 & 1 & &
\end{array}\right] .
$$

Case (2.1.2.1) Suppose that $a_{45}=1$. Then,

$$
A=\left[\begin{array}{llllll}
0 & 1 & 1 & 1 & 0 & \\
1 & 0 & 1 & 1 & 0 & \\
1 & 1 & 0 & 0 & 1 & \\
1 & 1 & 0 & 0 & 1 & \\
0 & 0 & 1 & 1 & 0 & 1 \\
& & & & 1 &
\end{array}\right]
$$

By repeated applications of Lemma 5.2 , we conclude that $A=Z(F, \ldots) \oplus A^{\prime}$, for some $A^{\prime}$ (possibly empty).

Case (2.1.2.2) Suppose that $a_{45}=0$. Then,

$$
A=\left[\begin{array}{llllll}
0 & 1 & 1 & 1 & 0 & 0 \\
1 & 0 & 1 & 1 & 0 & 0 \\
1 & 1 & 0 & 0 & 1 & 0 \\
1 & 1 & 0 & 0 & 0 & 1 \\
0 & 0 & 1 & 0 & 0 & \\
& & 0 & 1 & &
\end{array}\right]
$$

Therefore, $a_{56}=1$, otherwise $A[\{3,4,5,6\}]$ has the form $F_{S_{2}}$. Thus, by Theorem 2.4 and symmetry,

$$
A=\left[\begin{array}{lllllll}
0 & 1 & 1 & 1 & 0 & 0 & \\
1 & 0 & 1 & 1 & 0 & 0 & \\
1 & 1 & 0 & 0 & 1 & 0 & \\
1 & 1 & 0 & 0 & 0 & 1 & \\
0 & 0 & 1 & 0 & 0 & 1 & 1 \\
& & & 1 & 1 & 0 &
\end{array}\right]
$$


We have $a_{76}=1$, otherwise $A[\{4,5,6,7\}]$ has the form $F_{S_{2}}$. Thus, by symmetry,

$$
A=\left[\begin{array}{llllllll}
0 & 1 & 1 & 1 & 0 & 0 & & \\
1 & 0 & 1 & 1 & 0 & 0 & & \\
1 & 1 & 0 & 0 & 1 & 0 & & \\
1 & 1 & 0 & 0 & 0 & 1 & & \\
0 & 0 & 1 & 0 & 0 & 1 & 1 & \\
& 0 & 0 & 1 & 1 & 0 & 1 & \\
& & & & 1 & 1 & 0 & 1 \\
& & & & & & 1 &
\end{array}\right]
$$

Then, by repeated applications of Lemma 5.2 , we conclude that $A=Z(E, \ldots) \oplus A^{\prime}$, for some $A^{\prime}$ (possibly empty).

Case (2.2) Suppose that $a_{24}=0$. Then, by Theorem 2.4 and symmetry,

$$
A=\left[\begin{array}{lllll}
0 & 1 & 1 & 1 & 0 \\
1 & 0 & 1 & 0 & 1 \\
1 & 1 & 0 & & \\
1 & 0 & & & \\
0 & 1 & & &
\end{array}\right]
$$

If $a_{34}=1$, then $a_{35}=0$ and $A[\{2,3,4,5\}]$ has the form $F_{S_{1}}$. Thus, $a_{34}=0$ and, by Theorem 2.4 and symmetry, $A$ has the form

$$
A=\left[\begin{array}{llllll}
0 & 1 & 1 & 1 & 0 & \\
1 & 0 & 1 & 0 & 1 & \\
1 & 1 & 0 & 0 & 0 & 1 \\
1 & 0 & 0 & 0 & & \\
0 & 1 & 0 & & & \\
& & 1 & & &
\end{array}\right]
$$

Case (2.2.1) Suppose that $a_{45}=1$. Then $a_{46}=1$, otherwise $A[\{3,4,5,6\}]$ has the form $F_{S_{1}}$. Thus, by symmetry,

$$
A=\left[\begin{array}{llllll}
0 & 1 & 1 & 1 & 0 & \\
1 & 0 & 1 & 0 & 1 & \\
1 & 1 & 0 & 0 & 0 & 1 \\
1 & 0 & 0 & 0 & 1 & 1 \\
0 & 1 & 0 & 1 & 0 & \\
& & 1 & 1 & &
\end{array}\right]
$$


We have $a_{56}=1$, otherwise $A[\{2,4,5,6\}]$ has the form $F_{S_{2}}$. Therefore,

$$
A=\left[\begin{array}{llllll}
0 & 1 & 1 & 1 & 0 & 0 \\
1 & 0 & 1 & 0 & 1 & 0 \\
1 & 1 & 0 & 0 & 0 & 1 \\
1 & 0 & 0 & 0 & 1 & 1 \\
0 & 1 & 0 & 1 & 0 & 1 \\
0 & 0 & 1 & 1 & 1 & 0
\end{array}\right] \oplus A^{\prime}=Y \oplus A^{\prime}
$$

for some $A^{\prime} \in \mathcal{A}_{\text {sym }}^{0}(n-6,3)$.

Case (2.2.2) Suppose that $a_{45}=0$. Then, by Theorem 2.4 and symmetry,

$$
A=\left[\begin{array}{lllllllll}
0 & 1 & 1 & 1 & 0 & & & \\
1 & 0 & 1 & 0 & 1 & & & \\
1 & 1 & 0 & 0 & 0 & 1 & & \\
1 & 0 & 0 & 0 & 0 & 0 & 1 & 1 \\
0 & 1 & 0 & 0 & 0 & & & \\
& & 1 & 0 & & & & \\
& & & 1 & & & & \\
& & & 1 & & & &
\end{array}\right]
$$

We have $a_{57}=a_{58}=1$, otherwise $A[\{2,4,5,7\}]$ or $A[\{2,4,5,8\}]$ has the form $F_{S_{2}}$. Similarly, $a_{67}=a_{68}=1$, otherwise $A[\{3,4,6,7\}]$ or $A[\{3,4,6,8\}]$ has the form $F_{S_{2}}$. Thus,

$$
A=\left[\begin{array}{llllllll}
0 & 1 & 1 & 1 & 0 & 0 & 0 & 0 \\
1 & 0 & 1 & 0 & 1 & 0 & 0 & 0 \\
1 & 1 & 0 & 0 & 0 & 1 & 0 & 0 \\
1 & 0 & 0 & 0 & 0 & 0 & 1 & 1 \\
0 & 1 & 0 & 0 & 0 & 0 & 1 & 1 \\
0 & 0 & 1 & 0 & 0 & 0 & 1 & 1 \\
0 & 0 & 0 & 1 & 1 & 1 & 0 & 0 \\
0 & 0 & 0 & 1 & 1 & 1 & 0 & 0
\end{array}\right] .
$$

Since $A[\{4,6,7,8\}]$ has the form $F_{S_{3}}$, we obtain a contradiction.

Using Proposition 5.1 and the Remark 2.10 we obtain the following.

Corollary 5.4: If $A$ is a matrix of the form described in Theorem 5.3, $A$ is minimal for the Bruhat order on $\mathcal{A}_{\text {sym }}^{0}(n, 3)$. Consequently, the minimal matrices for the Bruhat order and for the Bruhat-graph order on $\mathcal{A}_{\mathrm{sym}}^{0}(n, 3)$ are the same.

\subsection{Maximal matrices}

We next give a class of maximal matrices for the Bruhat-graph order on $\mathcal{A}_{\text {sym }}^{0}(n, 3)$. We conjecture that there are no other maximal matrices beyond these. 
The matrix $A \in \mathcal{A}(n, 3)$ is maximal for the Bruhat order on $\mathcal{A}(n, 3)$ if and only if $L_{n} A$ is minimal for the Bruhat order on $\mathcal{A}(n, 3)$. Thus, taking into account Theorem 3.3 in [1], the maximal matrices for the Bruhat order on $\mathcal{A}(n, 3)$ are the skew-direct sums of $J_{3}, J_{4}-I_{4}$,

$$
W=L_{5} V=\left[\begin{array}{lllll}
0 & 0 & 1 & 1 & 1 \\
0 & 1 & 0 & 1 & 1 \\
1 & 0 & 0 & 1 & 1 \\
1 & 1 & 1 & 0 & 0 \\
1 & 1 & 1 & 0 & 0
\end{array}\right]
$$

$W^{\prime}=L_{5} V^{T}$, and $Q_{i}=L_{i+6} U_{i}, i \geq 1$, where, for $i \geq 1, U_{i}$ is the matrix in $\mathcal{A}^{S}(i+6,3)$ defined by

$$
U_{i}:=\left[\begin{array}{ccccccccc}
1 & 1 & 1 & 0 & & & & & \\
1 & 1 & 1 & 0 & & & & & \\
1 & 1 & 0 & 1 & & & & & \\
0 & 0 & 1 & 1 & 1 & & & & \\
& & & \ddots & \ddots & \ddots & & & \\
& & & & 1 & 1 & 1 & 0 & 0 \\
& & & & & 1 & 0 & 1 & 1 \\
& & & & & 0 & 1 & 1 & 1 \\
& & & & 0 & 1 & 1 & 1
\end{array}\right] .
$$

In particular,

$$
U_{1}=\left[\begin{array}{lllllll}
1 & 1 & 1 & 0 & 0 & 0 & 0 \\
1 & 1 & 1 & 0 & 0 & 0 & 0 \\
1 & 1 & 0 & 1 & 0 & 0 & 0 \\
0 & 0 & 1 & 1 & 1 & 0 & 0 \\
0 & 0 & 0 & 1 & 0 & 1 & 1 \\
0 & 0 & 0 & 0 & 1 & 1 & 1 \\
0 & 0 & 0 & 0 & 1 & 1 & 1
\end{array}\right]
$$

We will use the following additional matrices:

$$
Z=\left[\begin{array}{llllllll}
0 & 0 & 0 & 0 & 0 & 1 & 1 & 1 \\
0 & 0 & 0 & 0 & 0 & 1 & 1 & 1 \\
0 & 0 & 0 & 1 & 1 & 0 & 0 & 1 \\
0 & 0 & 1 & 0 & 1 & 0 & 1 & 0 \\
0 & 0 & 1 & 1 & 0 & 1 & 0 & 0 \\
1 & 1 & 0 & 0 & 1 & 0 & 0 & 0 \\
1 & 1 & 0 & 1 & 0 & 0 & 0 & 0 \\
1 & 1 & 1 & 0 & 0 & 0 & 0 & 0
\end{array}\right], \quad Z^{\prime}=\left[\begin{array}{llllllll}
0 & 0 & 0 & 0 & 0 & 1 & 1 & 1 \\
0 & 0 & 0 & 0 & 1 & 0 & 1 & 1 \\
0 & 0 & 0 & 1 & 0 & 0 & 1 & 1 \\
0 & 0 & 1 & 0 & 1 & 1 & 0 & 0 \\
0 & 1 & 0 & 1 & 0 & 1 & 0 & 0 \\
1 & 0 & 0 & 1 & 1 & 0 & 0 & 0 \\
1 & 1 & 1 & 0 & 0 & 0 & 0 & 0 \\
1 & 1 & 1 & 0 & 0 & 0 & 0 & 0
\end{array}\right]
$$

The following result can be easily verified using the definition of $\prec_{B}$.

Proposition 5.5: The matrices $Z$ and $Z^{\prime}$ are maximal for the Bruhat order on $\mathcal{A}_{\text {sym }}^{0}(8,3)$ and, therefore, for the Bruhat-graph order. 
The next result describes maximal matrices for the Bruhat-graph order on $\mathcal{A}_{\text {sym }}^{0}(n, 3)$.

Proposition 5.6: Let $n \geq 4$ be an even integer. A matrix in $\mathcal{A}_{\mathrm{sym}}^{0}(n, 3)$ is a maximal matrix for the Bruhat-graph order on $\mathcal{A}_{\text {sym }}^{0}(n, 3)$ if it is of the form

$$
A_{1}^{T} \oplus^{\prime} \cdots \oplus^{\prime} A_{r}^{T} \oplus^{\prime} A_{r} \oplus^{\prime} \cdots \oplus^{\prime} A_{1}, \quad r \geq 1,
$$

or

$$
A_{1}^{T} \oplus^{\prime} \cdots \oplus^{\prime} A_{r}^{T} \oplus^{\prime} B \oplus^{\prime} A_{r} \oplus^{\prime} \cdots \oplus^{\prime} A_{1}, \quad r \geq 0,
$$

where $A_{i} \in\left\{J_{3}, J_{4}-I_{4}, W, W^{\prime}, Q_{j}(j \geq 1)\right\}, i=1, \ldots, r$, and $B \in\left\{J_{4}-I_{4}, Z, Z^{\prime}\right\}$.

Proof: By Remark 2.5, the matrices $J_{4}-I_{4}, Z$ and $Z^{\prime}$ are maximal for the Bruhat-graph order. The matrices $J_{3}, J_{4}-I_{4}, W, W^{\prime}$ and $Q_{j}$, with $j \geq 1$ are maximal for the secondary Bruhat order. Using Lemma 2.9, the claimed skew-direct sum of them is maximal for the Bruhat-graph order.

Using Proposition 5.5 and Remark 2.10, we obtain maximal matrices for the Bruhat order on $\mathcal{A}_{\text {sym }}^{0}(n, 3)$.

Corollary 5.7: If $A$ is a matrix of the form described in Proposition 5.6, $A$ is maximal for the Bruhat order on $\mathcal{A}_{\mathrm{sym}}^{0}(n, 3)$.

We conjecture that $J_{4}-I_{4}, Z, Z^{\prime}$ are the only maximal matrices for the Bruhat-graph order on a class $\mathcal{A}_{\text {sym }}^{0}(n, 3)$ that cannot be written as skew-direct sums of smaller matrices. In this case, taking into account Proposition 5.5, they are the only maximal matrices for the Bruhat order on a class $\mathcal{A}_{\text {sym }}^{0}(n, 3)$ with this property. Thus, if our conjecture holds, the maximal matrices for the Bruhat-graph order and Bruhat order on $\mathcal{A}_{\text {sym }}^{0}(n, 3)$ coincide and are precisely the matrices given in Proposition 5.6.

\section{Conclusion}

We have studied the minimal and maximal matrices for the Bruhat and Bruhat-graph orders on the class of symmetric $(0,1)$-matrices with zero trace and a fixed row sum vector. We have given necessary conditions that should be satisfied by minimal and maximal matrices for these orders. In particular, we have shown that a minimal (maximal) matrix for the Bruhat-graph order may contain an anti-identity (identity) matrix of size 2 only if one of the zeros is on the main diagonal.

In the class of symmetric $(0,1)$-matrices with zero trace and constant row sum $k$, we characterized all the minimal matrices when $k=1,2,3$. We also presented the maximal matrices for $k=1,2$ and gave a class of such matrices for $k=3$ which we conjecture that includes all maximal matrices. In general, it may not be possible to characterize the extremal matrices for general $\mathcal{A}_{\text {sym }}^{0}(n, 4)$ in any satisfactory way. The following two examples give minimal matrices for $\mathcal{A}_{\text {sym }}^{0}(2 k, k)$ and $\mathcal{A}_{\text {sym }}^{0}(12,4)$. 
Example 6.1: The following is a minimal matrix for $\mathcal{A}_{\text {sym }}^{0}(2 k, k)$

$$
\left[\begin{array}{cc}
J_{k}-I_{k} & I_{k} \\
I_{k} & J_{k}-I_{k}
\end{array}\right] .
$$

Example 6.2: The following is a minimal matrix for $\mathcal{A}_{\text {sym }}^{0}(12,4)$

$$
D=\left[\begin{array}{ccccc}
0 & 1 & 1 & 1 & 1 \\
1 & 0 & 1 & 1 & 1 \\
1 & 1 & 0 & 1 & 1 \\
1 & 1 & 1 & 0 & 1 \\
1 & 1 & 1 & 1 & 0
\end{array}\right] \oplus\left[\begin{array}{lll|l|lll}
0 & 1 & 1 & 1 & 1 & 0 & 0 \\
1 & 0 & 1 & 1 & 1 & 0 & 0 \\
1 & 1 & 0 & 0 & 0 & 1 & 1 \\
\hline 1 & 1 & 0 & 0 & 0 & 1 & 1 \\
\hline 1 & 1 & 0 & 0 & 0 & 1 & 1 \\
0 & 0 & 1 & 1 & 1 & 0 & 1 \\
0 & 0 & 1 & 1 & 1 & 1 & 0
\end{array}\right]
$$

\section{Disclosure statement}

No potential conflict of interest was reported by the author(s).

\section{Funding}

This work was partially supported by the Fundação para a Ciência e a Tecnologia through the project UID/MAT/00297/2019. Also this work was partially supported by the Fundação para a Ciência e a Tecnologia through the project UID/MAT/04721/2019.

\section{References}

[1] Brualdi RA, Hwang S-G. A Bruhat order for the class of $(0,1)$-matrices with row sum vector $R$ and column sum vector $S$. Electron J Linear Algebra. 2004;12:6-16.

[2] Brualdi RA. Combinatorial matrix classes. Cambridge: Cambridge University Press; 2006. (Encyclopedia of Mathematics and its Applications; vol. 108).

[3] Brualdi RA, Deaett L. More on the Bruhat order for $(0,1)$-matrices. Linear Algebra Appl. 2007;421:219-232.

[4] da Cruz HF, Fernandes R, Furtado S. Minimal matrices in the Bruhat order for symmetric (0,1)-matrices. Linear Algebra Appl. 2017;530:160-184.

[5] Fernandes R, da Cruz HF, Salomão D. Classes of $(0,1)$-matrices where the Bruhat order and the secondary Bruhat order coincide. Order. 2019. doi:10.1007/s11083-019-09500-8.

[6] Ghebleh M. On maximum chains in the Bruhat order of $\mathcal{A}(n, 2)$. Linear Algebra Appl. 2014;446:377-387.

[7] Ghebleh M. Antichains on $(0,1)$-matrices through invertions. Linear Algebra Appl. 2014;458:503-511.

[8] Brualdi RA, Fernandes R, Furtado S. On the Bruhat order of labeled graphs. Discrete Appl Math. 2019;258:49-64. 